\title{
Sialoblastoma and Hepatoblastoma in an Infant: A Case Report and Review of the Literature
}

\author{
Lin $\mathrm{Xu}^{1}$ and Yumin Zhong, ${ }^{1 *}$ \\ ${ }^{1}$ Department of Radiology, Shanghai Children's Medical Center, School of Medicine, Shanghai Jiao Tong University, Shanghai, China \\ "Corresponding author: Department of Radiology, Shanghai Children's Medical Center, School of Medicine, Shanghai Jiao Tong University, Shanghai, China. Email: \\ zyumin2002@163.com
}

Received 2021 June 16; Revised 2021 September 26; Accepted 2021 September 26.

\begin{abstract}
Sialoblastoma is a rare salivary gland tumor, diagnosed in the neonatal period or during infancy. Hepatoblastoma accounts for less than $1 \%$ of all pediatric malignancies. The co-occurrence of these two tumors is extremely uncommon. Here, we report a case of concurrent sialoblastoma and hepatoblastoma in an infant.
\end{abstract}

Keywords: Sialoblastoma, Hepatoblastoma, AFP, CT, MRI

\section{Introduction}

Sialoblastoma is a rare primary salivary gland tumor in children, accounting for only 2 - 5\% of all childhood salivary gland tumors, with an annual incidence of 0.8 per million people. These tumors originate from the salivary gland epithelial cells (1-3). Hepatoblastoma is also a rare primary malignant liver tumor during infancy and childhood, which is responsible for less than $1 \%$ of all pediatric malignancies (4). The co-occurrence of these two tumors is exceptionally uncommon, and only few cases have been reported in the literature (5-7).

Here, we present a case of sialoblastoma with concomitant hepatoblastoma during infancy and reviewed the literature to summarize the presentations and imaging features.

\section{Case Presentation}

A five-month-old girl presented with a palpable mass on the left cheek, which had appeared after birth and progressively enlarged since the age of one month. A firm, nodular, non-tender mass $(5 \times 5 \mathrm{~cm})$ was palpable in the left parotid gland. The lesion was solid and did not fluctuate; the overlying skin was also normal. Abdominal examination revealed hepatomegaly $(4 \mathrm{~cm})$ with a hard right upper quadrant mass. The patient's surgical and family history was unremarkable. Blood investigations revealed that alpha-fetoprotein (AFP) was markedly elevated to more than $120,000 \mathrm{ng} / \mathrm{mL}$.

Contrast-enhanced computed tomography (CT) scan and magnetic resonance imaging (MRI) were also performed for the patient. The CT scan of the neck demonstrated a lobulated soft tissue mass with punctate calcifications in the left parotid. The lesion was hypodense to brain and isodense to muscle (Figure 1). Contrast-enhanced CT scan indicated heterogeneous enhancement. Besides, remodeling/thinning of the left maxillary, mandibular, and zygomatic bones were observed. However, the mass could not be well separated from the intraoral contents or the superficial neck muscles on CT scan. MRI revealed a wellcircumscribed lesion with septations in the left maxillofacial region (Figure 2). The tumor was isointense to muscle on T1-weighted images, with some hyperintense hemorrhagic areas. A slightly higher level of intermediate signal intensity was observed for the mass with some hyperintense necrotic areas in T2-weighted images. Diffusionweighted imaging (DWI) and apparent diffusion coefficient (ADC) indicated intense restricted diffusion.

Prominent heterogeneous enhancement was also detected on contrast-enhanced T1-weighted images. However, no cervical lymphadenopathy was reported in the patient. Also, the calvarium was normal without bone destruction. There was no evidence of an intracranial mass or abnormality. A large heterogenous lesion with lobulated margins in the liver was detected in the abdomi- 

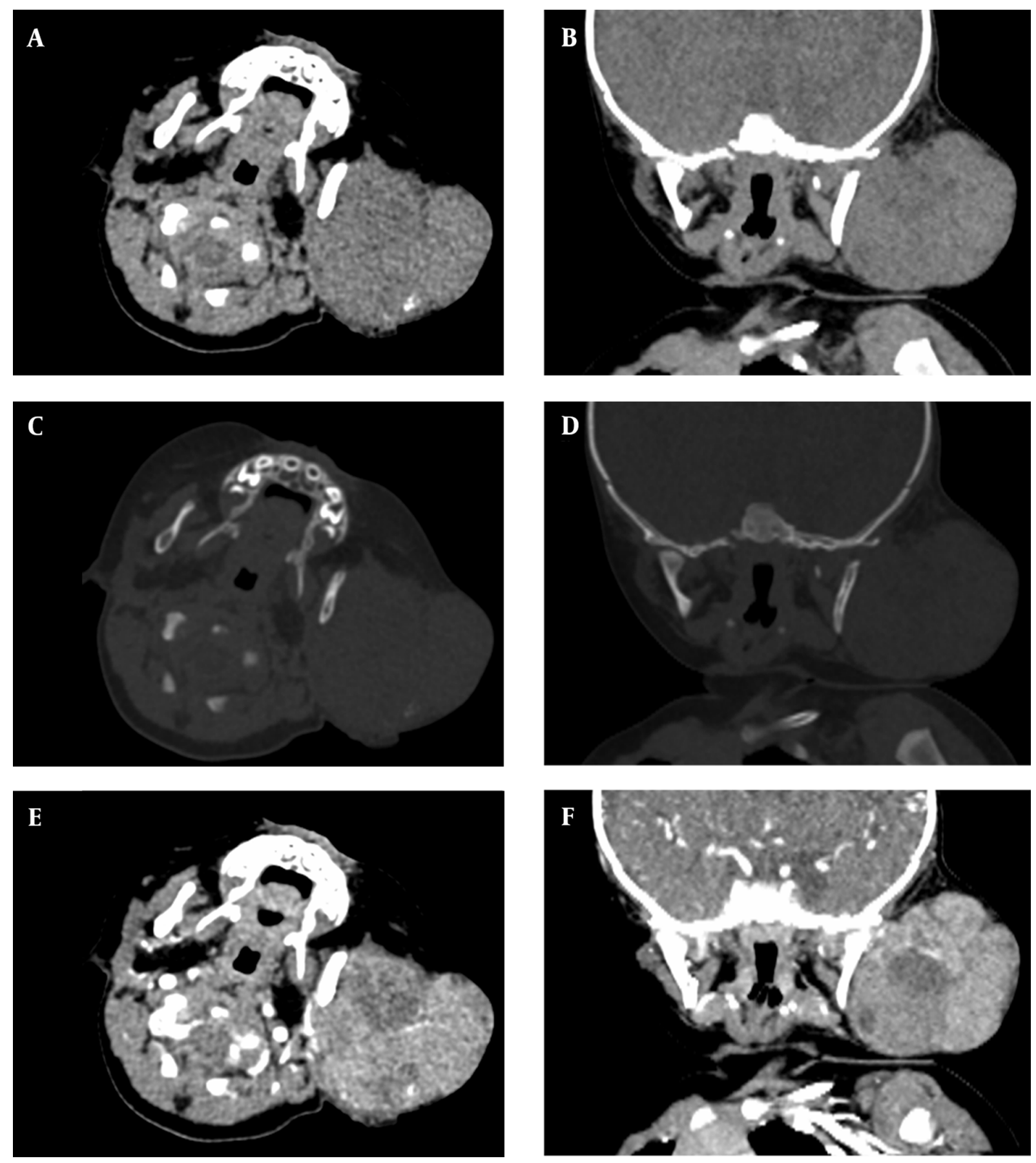

Figure 1. A five-month-old girl with a palpable mass on the left cheek associated with a hepatic mass. A lobulated soft tissue mass with punctate calcifications can be seen in the left cheek, A and B, Axial and coronal plain CT scans of the neck. C and D, Axial and coronal bone windows in the neck CT scans. Bone remodeling of the left ramus of the mandible can be seen; remodeling/thinning of the left maxillary and zygomatic bones is not shown; E and F, Axial and coronal contrast-enhanced CT scans show heterogeneous tumor enhancement. 

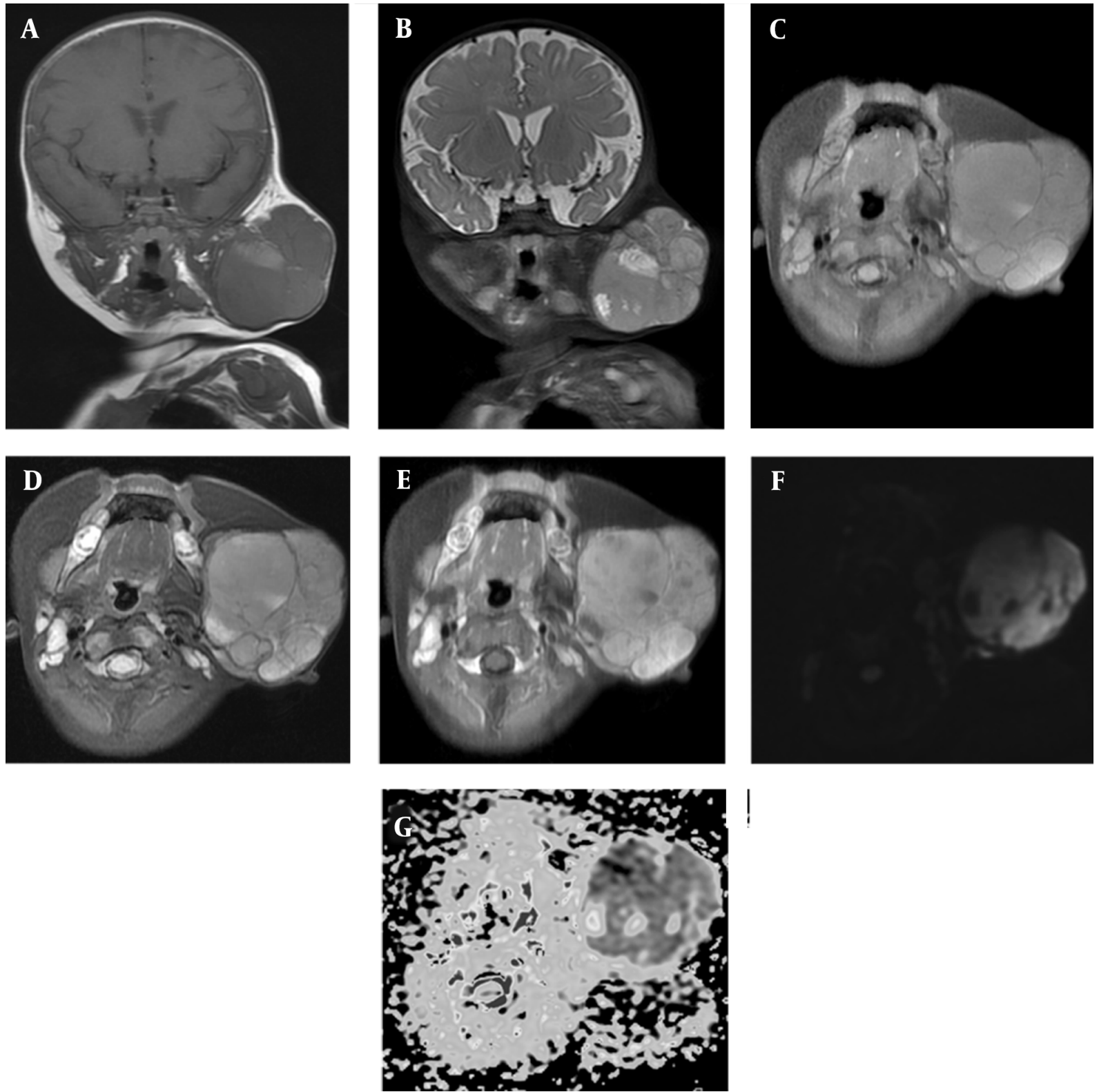

Figure 2. Contrast-enhanced MRI of the neck indicates a well-circumscribed lesion with hypointense septations in the left maxillofacial region. A and C, On coronal and axial T1-weighted images, tumor is isointense to muscle, with some hyperintense hemorrhagic areas; B and D, Coronal and axial T2-weighted images indicate slightly higher intermediate signal intensity with some hyperintense necrotic areas; E, Contrast-enhanced T1-weighted image indicates prominent heterogeneous enhancement; F and G, Diffusion-weighted image (DWI) and apparent diffusion coefficient (ADC) map represent intense restricted diffusion. 
nal MRI (Figure 3). The tumor showed low-intermediate signal intensity on T1-weighted images and a slightly higher intermediate signal intensity on T2-weighted images. The post-contrast sequences demonstrated moderate enhancement. The DWI and ADC also showed restricted diffusion. A differential diagnosis of salivary gland malignancy, possibly with a metastatic disease of the liver, was established.

Fine needle aspiration was performed for the left parotid, and histopathology revealed a malignant tumor, suggesting sialoblastoma. After several courses of chemotherapy, right lobar hemi-hepatectomy was performed in another hospital for the patient, and histopathology confirmed the diagnosis of hepatoblastoma.

\section{Discussion}

Sialoblastoma is a rare primary salivary gland tumor, which arises from the primitive duct epithelial cells. Some cases were detected by antenatal sonography or fetal MRI, while others were diagnosed by caregivers in the first few weeks of life. This type of neoplasm, which is located in the maxillofacial region, is three times more likely to occur in the parotid gland than in the submandibular gland $(2,8-$ 10).

The imaging features of sialoblastoma have been documented in a few cases. On CT scan, they are detected as lobulated lesions that are isodense to muscle $(11,12)$. On MRI, these tumors show low or intermediate signal intensity on T1-weighted images and intermediate or mildly high signal intensity on T2-weighted images, suggesting a high nucleus-to-cytoplasmic ratio (13-15). They are usually heterogeneous, with focal areas of necrosis or hemorrhage; these lesions may enhance poorly, heterogeneously, or intensely $(7,12,13)$. DWI and ADC measurements indicated intense restricted diffusion $(7,16)$. These tumors can be noninfiltrating or locally invasive into the adjacent muscle and bone. In our case, except for the typical imaging features of the lesion, hypointense separations and punctate calcifications were also observed in the mass.

Hepatoblastoma accounts for less than $1 \%$ of pediatric malignancies. It is estimated that two-thirds of tumors appear in the first two years of life. The serum AFP levels are markedly elevated in up to $90 \%$ of children with these tumors (4). On MRI, hepatoblastomas can be unifocal or multifocal with a typically low signal intensity on T1-weighted images and a heterogeneously high signal intensity on T2weighted images, along with varying degrees of hemor- rhage and necrosis. On post-contrast images, the lesion enhances heterogeneously and may demonstrate areas of early washout from arteriovenous shunting. Also, on DWI, hepatoblastomas exhibit intense diffusion restriction (17).

The co-occurrence of sialoblastoma and hepatoblastoma is extremely rare in infants. Only three cases have been reported in the English literature (Table 1). Because the liver and parotid glands are closely related embryonically, as they both develop from the foregut, it is postulated that an abnormality affecting the cells of these two organs can lead to two concurrent tumors in the child (6).

Generally, AFP is an important tumor marker in the evaluation of hepatic masses (4). In healthy neonates, it can be extremely high (e.g., 500,000 ng/mL), although it decreases by around the eighth month of life before reaching adult levels (18). Given the wide normal range of AFP during the first several months of life, it is challenging for clinicians to determine whether an individual's AFP level is abnormal and diagnose an AFP-producing tumor $(4,19)$. In our experience, the risk of hepatoblastoma is much higher if the AFP level exceeds $10,000 \mathrm{ng} / \mathrm{mL}$ in an infant with a hepatic mass above three months of age.

Apart from the abovementioned cases, a sialoblastoma in a neonate was associated with a high AFP level (39,032 $\mathrm{IU} / \mathrm{mL}$ ) (20). Tumor cells showed diffuse positive staining with AFP, and the staining density changed from slight to moderate. It can be postulated that sialoblastoma tumor cells also lead to elevated AFP levels in neonates, as the liver and parotid glands are closely related embryonically.

Hemangioma is the most common benign tumor during infancy, which can simultaneously occur in the salivary glands and liver (21). These lesions are well-defined masses, which can be unifocal or multifocal, with or without blue discoloration of the skin; the AFP level is typically normal. On MRI, the lesion is hypointense on T1-weighted images unless hemorrhage is present and hyperintense on T2-weighted images. In DWI, hemangioma typically exhibits no diffusion restriction. Centripetal contrast enhancement is the most specific imaging feature of hemangiomas $(17,21,22)$.

As shown in the present case, when an infant has a mass in the cheek with hypointense septations and punctate calcifications, and the APF level exceeds 10,000 ng/mL, sialoblastoma should be suspected, and abdominal imaging should be carried out. Overall, these findings with the aid of multiple imaging modalities can improve diagnostic certainty; besides, systemic and comprehensive treatment can lead to a successful patient recovery. 

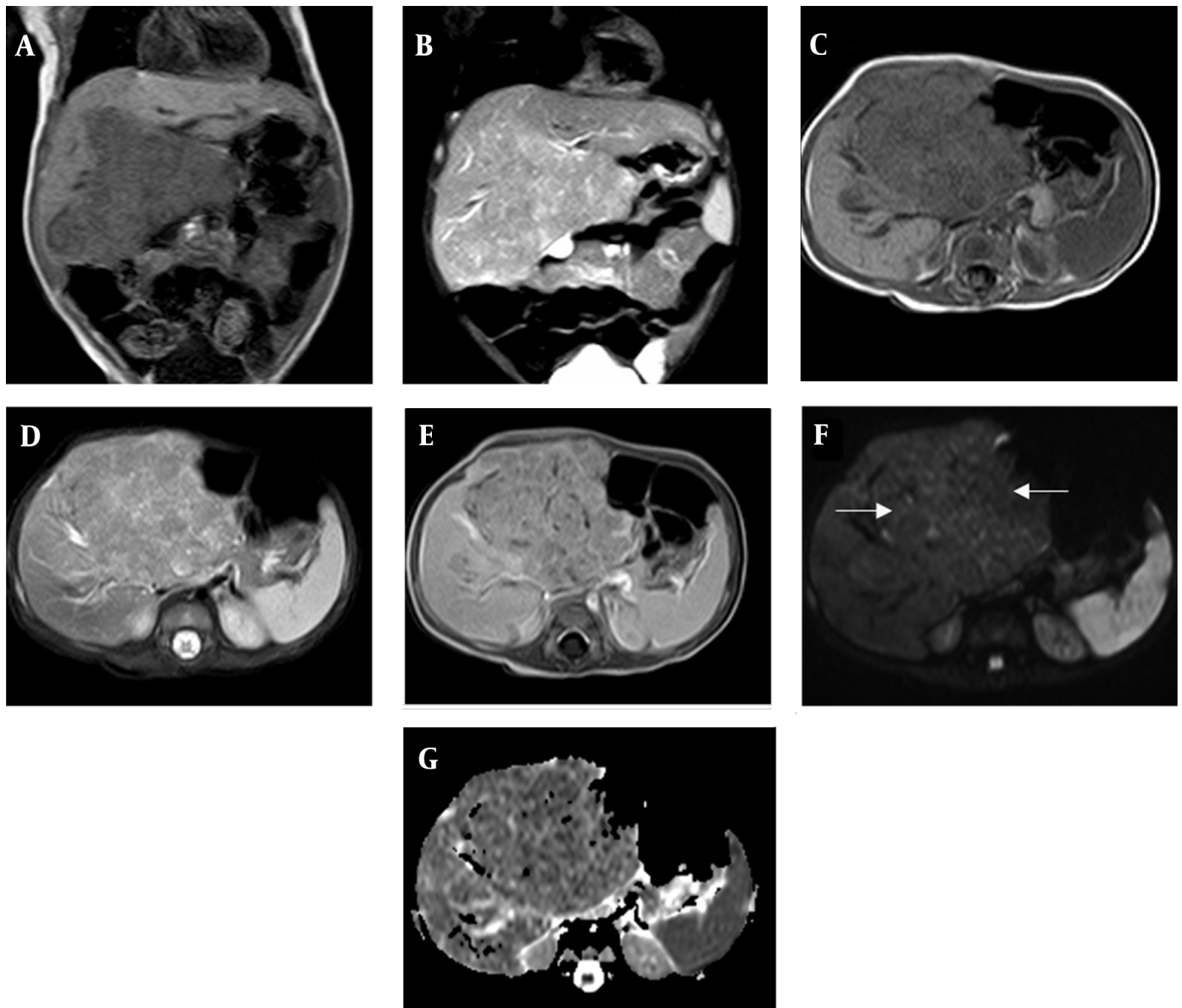

Figure 3. A large heterogeneous lesion with lobulated margins can be seen in the liver by contrast-enhanced MRI. A and C, Coronal and axial T1-weighted images; the tumor shows low to intermediate signal intensity; B and D, Coronal and axial T2-weighted images; the tumor shows slightly higher intermediate signal intensity; E, Post-contrast sequences show moderate enhancement; F and G, Diffusion-weighted image (DWI) and apparent diffusion coefficient (ADC) map indicate restricted diffusion (arrows).

\begin{tabular}{|c|c|c|c|c|c|}
\hline Year/reference & Age & Location of cheek lesions & AFP level (ng/mL) & Number of liver lesions & Imaging modalities \\
\hline $2000 /(5)$ & 37 weeks & Right parotid & 432,240 & Multiple & NK-CT/AB-MRI \\
\hline $2009 /(6)$ & 34 weeks & Right parotid & NA & Multiple & NK-US/AB-US \\
\hline $\mathbf{2 0 1 2} /(7)$ & 37 weeks & Left parotid & 671,500 & Solitary & Fetal US and MRI/NK-MRI/AB-US and CT \\
\hline
\end{tabular}

Abbreviations: AB, abdomen; AFP, alpha fetoprotein; CT, computed tomography; MRI, magnetic resonance imaging; NA, not available; NK, neck; US, ultrasound.

\section{Footnotes}

Authors' Contribution: Lin Xu substantially contributed to the conception and design of the manuscript. Lin $\mathrm{Xu}$ and Yu-Min Zhong were involved in drafting the manuscript and its critical revision. Both authors participated sufficiently in the work to take public responsibility for the content. Both authors also agreed to be accountable for all aspects of the work in ensuring that questions related to the accuracy or integrity of any part of the work 
are appropriately investigated and resolved. Both authors read and approved the final manuscript for publication.

Conflict of Interests: The authors have no affiliation with any organization with a direct or indirect financial interest in the subject matter discussed in the manuscript.

Funding/Support: This study received no funding/support.

Informed Consent: Written informed consent was obtained from the patient

\section{References}

1. Wang Q, Ma S, Chen H, Yang M, Cai W. Sialoblastoma in chin and management of treatment. Int J Pediatr Otorhinolaryngol. 2018;109:168-73. doi:10.1016/j.ijporl.2018.03.023. [PubMed: 29728174].

2. Irace AL, Adil EA, Archer NM, Silvera VM, Perez-Atayde A, Rahbar R. Pediatric sialoblastoma: Evaluation and management. Int J Pediatr Otorhinolaryngol. 2016;87:44-9. doi: 10.1016/j.ijporl.2016.04.037. [PubMed: 27368441].

3. Choudhary K, Panda S, Beena VT, Rajeev R, Sivakumar R, Krishanan S. Sialoblastoma: A literature review from 1966-2011. Natl J Maxillofac Surg. 2013;4(1):13-8. doi: 10.4103/0975-5950.117821. [PubMed: 24163547]. [PubMed Central: PMC3800378].

4. Chavhan GB, Siddiqui I, Ingley KM, Gupta AA. Rare malignant liver tumors in children. Pediatr Radiol. 2019;49(11):1404-21. doi: 10.1007/s00247-019-04402-8. [PubMed: 31620842].

5. Siddiqi SH, Solomon MP, Haller JO. Sialoblastoma and hepatoblastoma in a neonate. Pediatr Radiol. 2000;30(5):349-51. doi: 10.1007/s002470050758. [PubMed: 10836603].

6. Stones DK, Jansen JC, Griessel D. Sialoblastoma and hepatoblastoma in a newborn infant. Pediatr Blood Cancer. 2009;52(7):883-5. doi: 10.1002/pbc.21945. [PubMed: 19156857].

7. Cheng YK, Chu WC, Law LW, Ting YH, Leung TY. A fetus with a huge neck mass and a large abdominal circumference-a rare case of sialoblastoma and hepatoblastoma. Prenat Diagn. 2012;32(9):915-7. doi: 10.1002/pd.3927. [PubMed: 22711473].

8. Di Micco R, Prufer F, Bruder E, Schifferli A, Gurtler N. Sialoblastoma of the submandibular gland: A distinct entity? Eur J Pediatr. 2019;178(8):1301-4. doi: 10.1007/s00431-019-03411-x. [PubMed: 31257547].

9. Ersoz S, Turgutalp H, Cobanoglu U, Bektas D, Yaris N. Sialoblastoma in the parotid gland: A case report. Pediatr Int. 2010;52(4):670-2. doi: 10.1111/j.1442-200X.2010.03108.x. [PubMed: 20958880].
10. Vidyadhar M, Amanda C, Thuan Q, Prabhakaran K. Sialoblastoma J Pediatr Surg. 2008;43(10):e11-3. doi: 10.1016/j.jpedsurg.2008.04.035. [PubMed: 18926196].

11. Sitthichaiyakul P, Somran J, Oilmungmool N, Worasakwuttipong S, Larbcharoensub N. Sialoblastoma of the cheek: A case report and review of the literature. Mol Clin Oncol. 2016;4(6):925-8. doi: 10.3892/mco.2016.840. [PubMed: 27284424]. [PubMed Central: PMC4887838].

12. Saravakos P, Hartwein J, Fayyazi A. Sialoblastoma of the parotid gland in a 13-year-old girl with multiple recurrences and long-term followup. Head Neck. 2016;38(1):E13-5. doi: 10.1002/hed.24084. [PubMed: 25900370].

13. Som PM, Brandwein M, Silvers AR, Rothschild MA. Sialoblastoma (embryoma): MR findings of a rare pediatric salivary gland tumor. AJNR Am J Neuroradiol.1997;18(5):847-50. [PubMed: 9159361]. [PubMed Central: PMC8338107].

14. Yekeler E, Dursun M, Gun F, Kilincaslan H, Ucar A, Genchellac H, et al. Sialoblastoma: MRI findings. Pediatr Radiol. 2004;34(12):1005-7. doi 10.1007/s00247-004-1286-5. [PubMed: 15278323].

15. Kataria SP, Kumar S, Singh G, Kalra R, Sen R, Garg N. Sialoblastoma. diagnosis by FNAC: A case report. Diagn Cytopathol. 2015;43(11):924-7. doi: 10.1002/dc.23324. [PubMed: 26214398].

16. Papaioannou G, Sebire NJ, McHugh K. Imaging of the unusual pediatric 'blastomas'. Cancer Imaging. 2009;9:1-11. doi: 10.1102/14707330.2009.0001. [PubMed: 19237343]. [PubMed Central: PMC2651735].

17. Masand PM. Magnetic resonance imaging features of common focal liver lesions in children. Pediatr Radiol. 2018;48(9):1234-44. doi: 10.1007/s00247-018-4218-5. [PubMed: 30078045].

18. Schieving JH, de Vries M, van Vugt JM, Weemaes C, van Deuren M, Nicolai J, et al. Alpha-fetoprotein, a fascinating protein and biomarker in neurology. Eur J Paediatr Neurol. 2014;18(3):243-8. doi: 10.1016/j.ejpn.2013.09.003. [PubMed: 24120489].

19. Ng K, Mogul DB. Pediatric Liver Tumors. Clin Liver Dis. 2018;22(4):75372. doi: 10.1016/j.cld.2018.06.008. [PubMed: 30266161].

20. Ozdemir I, Simsek E, Silan F, Demirci F. Congenital sialoblastoma (embryoma) associated with premature centromere division and high level of alpha-fetoprotein. Prenat Diagn. 2005;25(8):687-9. doi: 10.1002/pd.1224. [PubMed: 16049999].

21. Vilanova JC, Barcelo J, Smirniotopoulos JG, Perez-Andres R, Villalon M, Miro J, et al. Hemangioma from head to toe: MR imaging with pathologic correlation. Radiographics. 2004;24(2):367-85. doi: 10.1148/rg.242035079. [PubMed: 15026587].

22. Inarejos Clemente EJ, Navallas $M$, Tolend $M$, Sunol Capella M, RubioPalau J, Albert Cazalla A, et al. Imaging Evaluation of Pediatric Parotid Gland Abnormalities. Radiographics. 2018;38(5):1552-75. doi 10.1148/rg.2018170011. [PubMed: 30096049]. 